\title{
STUDIO DELLA PROTEZIONE COLLETTIVA SUI DIRITTI DI SOLIDARIETÀ
}

\section{ARTICOLO ORIGINALE}

RIBEIRO, Danilo Miranda ${ }^{1}$

RIBEIRO, Danilo Miranda. Studio della protezione collettiva sui diritti di solidarietà. Revista Científica Multidisciplinar Núcleo do Conhecimento. Anno. 06, Ed. 12, Vol. 02, pp. 152-167. Dicembre 2021. ISSN: 2448-0959, Link di accesso: https://www.nucleodoconhecimento.com.br/legge/diritti-di-solidarieta, DOI: 10.32749/nucleodoconhecimento.com.br/legge/diritti-di-solidarieta

\section{RIEPILOGO}

Questo lavoro cerca di costruire un'analisi legale sugli elementi del processo collettivo per la protezione dei diritti di solidarietà nel sistema giuridico brasiliano. Poiché le sue disposizioni nelle leggi sono distanziate, lo studio della protezione collettiva è il problema di un sistema ermetico difficile da capire. Con l'obiettivo di far posto allo studio del processo collettivo e di escludere questo problema, questa ricerca ha come problematica la discussione sui principali elementi del processo collettivo, con l'obiettivo di dimostrare che si tratta di un sistema procedurale coerente e armonioso. La metodologia utilizzata sarà la revisione bibliografica. Pertanto, il lavoro presenta come risultato la sistematizzazione di elementi fondamentali alla comprensione della protezione collettiva, quando si affrontano le disposizioni legali e dottrinali sulla protezione dei diritti di solidarietà, al fine di aprire il campo per una comprensione della protezione collettiva.

Parole chiave: Processo collettivo, diritti di solidarietà, Procedura civile, Principi.

\footnotetext{
${ }^{1}$ Master in Diritti Umani e Democrazia presso I'UFPR-Università Federale del Paraná. Laurea in Giurisprudenza presso I'UCSal-Università Cattolica di Salvador. ORCID: https://orcid.org/0000-0001-6503-9625.
}

RC: 103912

Disponibile in: https://www.nucleodoconhecimento.com.br/legge/diritti-di-solidarieta 


\section{INTRODUZIONE}

La protezione collettiva è uno strumento procedurale essenziale per la realizzazione della giustizia sociale attraverso la protezione di beni giuridici che non appartengono solo a un individuo, ma riguardano la collettività. Si scopre che le disposizioni legali sull'istituto si trovano in una legislazione varia e non rinunciano alla comprensione costituzionale della questione, che può essere un ostacolo all'indagine coerente del processo collettivo. In considerazione di questo problema, questa ricerca mira a offrire una riflessione sugli elementi essenziali della protezione collettiva dei diritti di solidarietà, come il concetto di diritti di solidarietà, legittimità e competenza. Pertanto, il processo collettivo sarà presentato in modo coerente e armonioso con i principi costituzionali guida, al fine di rimuovere l'ermetismo che può essere percepito conoscendo la protezione collettiva. La metodologia utilizzata sarà la revisione bibliografica degli autori che hanno dato un importante contributo alla comprensione della tutela dei diritti di solidarietà.

Dal punto di vista costituzionale, il principio del giusto processo (art. 5, LIV, CF/88) deve essere interpretato nella sua dimensione collettiva, poiché mira a ridurre le formalità al fine di fornire un processo meta-individuale effettivo ed equo alla giurisdizione. II sistema processuale collettivo attribuisce legittimità a soggetti diversi (ope legis), poiché la rilevanza degli interessi tutelati evoca la responsabilità dei diversi attori di garantire i diritti di una collettività. In questo senso, l'art. 103, del CDC pone l'efficacia erga omnes o ultra partes per le decisioni emesse nel processo collettivo (BELLINETTI; SOUZA, 2019).

Per quanto riguarda la classificazione, Zavascki (2005, p. 18) divide gli interessi collettivamente in due categorie. II primo sarebbero interessi individuali omogenei, che avrebbero il loro carattere collettivo per una questione empirica, di contingenza, non necessaria, in cui ci sarebbero più soggetti, ma l'oggetto è divisibile. La seconda categoria riguarda gli interessi diffusi e collettivi, costituiti da quelli che sono collettivi per la loro essenza, che riguardano un oggetto indivisibile e un numero incerto di 
soggetti. In relazione agli interessi solidale, è possibile concepire in due aree separate: diritti individuali omogenei e diritti collettivi stricto sensu e diffusi.

Secondo Ronaldo Santos (2014), l'idea di proteggere il diritto collettivo è garantire la giustizia sociale per i gruppi sociali offesi o legalizzare situazioni che prima non avevano protezione giudiziaria. Per l'autore, l'azione collettiva ha avuto come antecedente il Bill of Peace, che risale alla storia del XVII secolo:

[...] tratava de uma autorização para o processamento de uma ação individual sob a forma coletiva, concedida nas hipóteses em que o autor requeria que o provimento da demanda englobasse os direitos de todos os indivíduos que estivessem em igual condição de litígio, para que a questão fosse tratada de modo uniforme e com vistas a evitar a multiplicação de processos (SANTOS, 2014, p. 238).

Con un'enfasi sul rapporto tra i diritti di solidarietà come diritti fondamentali, Hachem comprende che i primi portano gli stessi elementi che sarebbero legati ai diritti fondamentali delle tre generazioni, non solo della terza, seconda o prima generazione:

Por conta da complexidade de sua natureza jurídica e estrutura normativa, todos os direitos fundamentais reúnem concomitantemente a totalidade dos traços que supostamente seriam peculiares a cada uma das gerações: (i) impõem deveres negativos ao Estado; (ii) dirigem ao Poder Público obrigações de fornecer prestações fáticas e normativas; (iii) ostentam a titularidade transindividual alegadamente exclusiva dos "direitos de terceira geração", bem como, simultaneamente, a titularidade individual pretensamente típica dos "direitos de primeira e segunda geração" (HACHEM, 2013, p. 621).

Attraverso le richieste collettive, l'accesso alla giustizia di una collettività indeterminata $\mathrm{o}$ di un determinato gruppo di persone è amplificato. La socializzazione e la democratizzazione del processo sono indicate come benefici di queste azioni, con la realizzazione dei diritti e l'alterazione della realtà sociale; la possibilità di correzione di un illecito che non sarebbe postulato in una domanda individuale prima del suo piccolo valore particolare, ma che collettivamente ha un grande impatto sociale; uguaglianza tra le parti in causa; la molecolarizzazione della domanda e la realizzazione del principio della certezza del diritto e del legittimo 
affidamento di fronte a decisioni uniformi per casi analoghi; la riduzione delle richieste nel sistema giudiziario, tra molti altri.

Più specificamente, gli interessi individuali omogenei indossano l'abbigliamento degli interessi individuali, ma di fronte a certe configurazioni della società, c'è bisogno di essere protetti collettivamente. La nomenclatura stessa traduce già parte del concetto di questo interesse, secondo la lezione di Zavascki:

$\mathrm{Na}$ verdade, a própria denominação interesses individuais homogêneos já exibe dois enunciados: de um lado, trata-se de interesses ontologicamente individuais, não coletivos; de outro lado, que consistem numa multiplicidade de interesses individuais apresentando um determinado grau de uniformidade ou homogeneidade, sua característica mais importante (ZAVASCKI, 2005, p. 18).

Ai sensi dell'articolo 81 della legge 8.078/91, gli interessi individuali omogenei si basano sull'idea di origine comune, sul fatto dannoso che ha colpito e violato innumerevoli persone nella loro individualità, senza alcun requisito di un rapporto giuridico di base tra i titolari o tra le parti, né un'unità di fatto o temporalità (ZAVASCKI, 2005, p. 21).

Gli interessi individuali omogenei sono quelli che hanno nella loro origine un fatto dannoso che ha inciso su una collettività e supera la sfera dell'interesse individuale. Pertanto, questi diritti hanno un'origine comune, che sorgono in comportamenti omissivi o commissivi della parte avversa che ledono diritti che sfuggono al mero aspetto individuale e creano la pretesa collettiva, portando alla discussione questioni superiori al singolo mero lide.

Secondo la lezione di Zavascki (2005, p. 21), sono divisibili, appropriati individualmente, disponibili, trasferibili e rappresentati in tribunale dal titolare stesso, episodicamente solidale, soggettivamente determinati e direttamente riparabili.

Tuttavia, la caratteristica dell'individualità non toglie l'eminente contenuto solidale dei diritti individuali omogenei. Se considerati collettivamente, questi diritti vanno ben oltre la sfera dell'interesse privato dei loro titolari. La caratteristica collettiva non 
risiede nell'impossibilità di appropriarsi individualmente o nell'indeterminabilità dei loro titolari, ma nella portata che la violazione rivela alla società (ZAVASCHI, 2005, p. 21-22). II danno agli interessi individuali omogenei viola la pace sociale e promuove un grande disagio sociale, che genera un effetto sinergico, poiché entrambi sono rafforzati.

Sarebbe anche possibile nominare interessi individuali rilevanti o interessi comuni, cosa che non è stata fatta perché l'omogeneità è essenziale per l'opportunità di proteggere collettivamente la legge (ZAVASCKI, 2005, p. 19).

Anche in relazione ai diritti di solidarietà, sorgono le categorie di diritti diffusi e collettivi stricto sensu. In primo luogo, i diritti diffusi sono quegli interessi collettivi che sono indivisibili, i cui titolari sono indeterminabili e non hanno un rapporto giuridico tra loro (DIDIER JUNIOR; ZANETI JUNIOR, 2014, p. 76-78). II concetto di diritto diffuso si ritrova analizzando l'art. 81, I, del Codice del Consumo, che sono concettualizzati come interessi solidale di natura indivisibile, i cui titolari sono indeterminati e collegati da un fatto comune.

I diritti diffusi sono quelli che appartengono a una collettività di soggetti indeterminati, portatori di diritti indivisibili e legati da una situazione di fatto (superveniente). Cioè, il rapporto tra i detentori è sorto in un momento successivo alla lesione, poiché non vi era alcun legame tra il ferito in un momento precedente al fatto che ha dato causa alla protezione collettiva (DIDIER JÚNIOR; ZANETI JÚNIOR, 2014, p. 78).

Poi ci sono i diritti collettivi stricto sensu, che trovano definizione nell'art.81, II del CDC. Sono diritti di solidarietà, con la differenza che i loro titolari sono determinati gruppi definiti di persone, cioè esiste un rapporto giuridico di base tra i titolari, che sono indeterminati ma determinabili. Cioè, la differenza tra diritti diffusi e collettivi stricto sensu è che i primi hanno una proprietà indeterminata e i secondi hanno determinate categorie collettive come titolari, quindi le persone possono essere determinate (DIDIER JÚNIOR; ZANETI JÚNIOR, 2014, p. 78-79). 
Per gli interessi collettivi stricto sensu è essenziale identificare quale gruppo di individui è stato danneggiato, poiché il diritto è indivisibile e i titolari sono coloro che appartengono a una certa categoria (DIDIER JÚNIOR; ZANETI JÚNIOR, 2014, p. 78).

Vale la pena notare che il rapporto giuridico di base dovrebbe essere precedente all'evento dell'evento dannoso. Cioè, il rapporto giuridico tra i titolari non deriva dal danno, come avviene nei diritti diffusi, ma a causa dell'adesione alla categoria del lavoro, all'associazione, a determinate società e ad altre cause che hanno preceduto il danno (DIDIER JÚNIOR; ZANETI JÚNIOR, 2014, p. 78). In questi modi, i titolari del diritto sono pienamente determinabili e appartengono a una collettività che è individualizzabile.

\section{LEGITTIMITÀ}

In situazioni di singolare giurisdizione, quasi sempre, gli argomenti in questione sono di interesse solo per le parti coinvolte nel corso, senza avere alcun peso diretto su persone che non sono coinvolte nel polo passivo o attivo del corso.

Tradizionalmente, la legittimità attiva della protezione dell'interesse individuale richiede un esame della proprietà e risale all'eredità del diritto romano (DIDIER JÚNIOR; ZANETI JÚNIOR, 2014, p. 79). D'altra parte, l'istituto del diritto in nome di un'altra persona non è qualcosa di molto più attuale, poiché "[...] nel Medioevo, non esisteva ancora il concetto di personalità giuridica, quindi non era nemmeno possibile considerare che qualcuno fosse rappresentato da un'altra persona; la legittimità ordinaria era la regola assoluta" (ZAVASCKI, 2005, p. 11).

Nell'ordinamento giuridico brasiliano, la regola generale è prevista dall'articolo $18 \mathrm{del}$ codice di procedura civile, che pone la proprietà del diritto come requisito fondamentale per la sua discussione, poiché rimane la discussione del diritto che appartiene alla persona di un altro, fatti salvi i casi espressamente previsti dalla legge. 
Tuttavia, oltre alla legittimità ordinaria, rappresentata dall'articolo 18 del CPC, esiste, in via eccezionale, una straordinaria legittimità attiva, ammessa nei casi espressamente previsti dalla legge, in cui qualcuno farà valere il diritto per conto del titolare. In questo caso, esiste un'opzione legislativa sui casi in cui sarà possibile invocare il diritto di altri in tribunale.

Al di là dei casi previsti dall'organo giuridico, le ipotesi di legittimità straordinaria devono essere considerate come una logica conseguenza dell'ordinamento giuridico. Neves (2016) è uno degli autori che commentano la possibilità di una legittimità straordinaria in assenza di un'espressa autorizzazione legale:

Apesar de o art. 18, caput, do Novo CPC prever que a legitimação depende de autorização expressa da lei, a melhor doutrina entende que, além da previsão legal, também se admite a legitimação extraordinária quando decorrer logicamente do sistema, como acontece com a legitimação recursal do advogado em apelar do capítulo da sentença que versa sobre seus honorários advocatícios (NEVES, 2016, p. 188).

La flessibilità in relazione alla regola dell'articolo 18 del CPC appare come un modello migliore per l'efficacia dell'istituto, proprio per difendere gli interessi della collettività. Tuttavia, è avvertito: la flessibilità non comporta come inevitabile follow-up una procedura più semplice.

È evidente che lo stesso diritto può essere garantito a persone di diversi gruppi sociali, che si verifica con una certa frequenza, ad esempio quando vi è una violazione delle garanzie sociali. Pertanto, vi è una difficoltà nell'affermare che esiste una legittimità a rappresentare tutti gli interessati, in considerazione della grande differenza culturale, sociale o economica che esiste tra i titolari dei diritti nelle richieste collettive.

È in questo contesto (ZAVASCKI, 2005, p. 15) che sono iniziate le difficoltà di difendere gli interessi collettivi. Se gli individui sono diversi, come si fa a presumere che uno di loro abbia la legittimità di chiedere a tutti in difesa di questo interesse comune? Come si possono estendere gli effetti della protezione collettiva su coloro che non sono in tribunale se non si può presumere quale sia l'interesse legittimo di 
questi altri? Come pretende che grandi gruppi compaiano in tribunale? Come fare se i membri del gruppo non sono nemmeno determinati?

In considerazione dell'indeterminazione dei titolari, di una grande differenza che esiste tra loro e della difficoltà pratica di tutti di portare determinate questioni in tribunale, la legittimazione di entità che hanno come scopo è la ricerca e la difesa degli interessi di una collettività.

In questo modo, i legittimati collettivi non sono titolari dei diritti che sono legittimati a perorare e i titolari di tali diritti hanno la proprietà attiva svuotata (NEVES, 2016, p. 189). Per eccellenza, nella lettera di Zavascki (2005, p. 15), la legittimità attiva nelle rivendicazioni collettive potrebbe essere nominata come la rappresentazione degli assenti.

Nella giurisdizione collettiva, non esiste un legame diretto tra la proprietà del diritto e la legittimità, ma esiste una situazione giuridica che porta la capacità di alcune istituzioni a difendere interessi che non si limitano all'idea di individualismo, che porta la configurazione di una legittimità straordinaria. In questo senso:

A legitimação ao processo coletivo é extraordinária: autoriza-se um ente a defender, em juízo, situação jurídica de que é titular um grupo ou uma coletividade. Não há coincidência entre o legitimado e o titular da situação jurídica discutida (DIDIER JUNIOR; ZANETI JUNIOR, 2014, p. 21).

II sistema giuridico brasiliano ha accettato l'opzione di assumere la capacità di alcune istituzioni di essere legittimate per difendere le richieste collettive (ZAVASCKI, 2005, p. 102). Cioè, quelli legittimati ai fini delle azioni collettive derivano dall'istituzione legale, come accade nell'articolo 5 della legge sull'azione civile pubblica, che elenca la Procura della Repubblica, I'Ufficio del difensore pubblico, I'Unione federale, gli Stati, il Distretto federale, i Comuni e altri soggetti, come rappresentanti della collettività e in grado di presentare azioni civili pubbliche.

Nello stesso movimento del LACP, l'articolo 82 del Codice della tutela del consumo evidenzia la legittimità attiva della Procura della Repubblica, dell'Unione, degli Stati e 
dei Comuni, dei comuni, delle società pubbliche, delle fondazioni, delle società o associazioni a economia mista a difendere i diritti omogenei diffusi, collettivi o individuali dei consumatori.

Inoltre, in relazione alla legittimità attiva ai fini delle azioni collettive, Zavascki (2005, pag. 132) solleva un aspetto controverso che circonda la possibilità che la Procura della Repubblica difenda interessi individuali omogenei, poiché il CRFB/88 non conferisce tale funzione al parquet.

II vernacolo costituzionale, nel suo articolo 129, punto III, non elenca la difesa di interessi individuali omogenei come una delle attribuzioni della Procura della Repubblica, che ha le sue attività legate al testo costituzionale. Pertanto, è possibile interpretare come un recinto giuridico la mancanza di determinazione a difendere tali interessi (ZAVASCKI, 2005, pag. 132).

Altri sostengono la legittimità illimitata della Procura della Repubblica (ZAVASCKI, 2005, p. 133). Ai sensi dell'articolo 129, punto IX, del CRFB/88, è compito del MP svolgere funzioni compatibili con il suo scopo, che include la difesa degli interessi sociali e individuali non disponibili, alla luce dell'articolo 127, il CRFB/88. Per questa corrente, gli interessi solidale hanno una presunzione di rilevanza sociale, che consente al parquet di agire (ZAVASCKI, 2005, p. 133).

Infine, c'è la tesi della legittimità ristretta, che emerge come conseguenza dell'idea che il pubblico ministero avrebbe una legittimità illimitata, con l'avvertimento che le questioni di rilevanza sociale potrebbero essere solo quelle che contengono congruenze con le funzioni costituzionali del parquet (ZAVASCKI, 2005, p. 132), questo basato sull'art. 127, CRFB/88, senza lo sforzo di presumere la rilevanza per poter legittimare il pubblico ministero.

Sulla legittimità della Procura della Repubblica nella tutela di interessi individuali omogenei, Zavascki conclude che la risposta a questa domanda sta attraverso l'analisi dei costumi giuridici, perché è nella legge che risiede il concetto di interesse di rilevanza sociale, che garantirà la legittimità attiva del parquet (ZAVASCKI, 2005, 
p. 132). In questo modo, Zavascki insegna che gli interessi di rilevanza sociale sono costituiti da: "imposizione di indisponibilità, l'attribuzione diretta di legittimità alla Procura della Repubblica per la sua difesa e l'espressa caratterizzazione della rilevanza sociale" (ZAVASCKI, 2005, p. 136).

La scissione è incontrovertibile. In assenza dell'aspetto di rilevanza sociale ritratto da Zavascki, non c'è bisogno di parlare di legittimità del parquet ad agire in difesa di interessi individuali omogenei.

Infine, vale la pena evidenziare le caratteristiche che Didier rivela nella legittimazione collettiva attiva, come regolamento dell'articolo 5 della legge federale n. 7.347/85 e dell'articolo 82 del $C D C$, essendo portato da entità che non agiscono in nome proprio, poiché i titolari non hanno capacità postulatoria:

Enfim, o certo é que a legitimação coletiva possui as seguintes características: a) está regulada, inicialmente, por lei (art. 5º da Lei Federal no 7.347/85; art. 82 do CDC etc.); b) é conferida a entes públicos, privados e despersonalizados, e, até, ao cidadão, na ação popular; c) o legitimado coletivo atua em nome próprio na defesa de direitos que pertencem a um agrupamento humano (pessoas indeterminadas, comunidade, coletividade ou g rupo de pessoas, titulares de direitos individuais abstratamente considerados, na forma do a1i. 81 do CDC e seus incisos); d) esse agrupamento humano não tem personalidade judiciária, portanto não pode atuar em juízo para proteger os seus direitos, 26 cuja defesa cabe aos legitimados coletivos, que possuem legitimação autônoma, exclusiva e concorrente e simples (DIDIER JUNIOR; ZANETI JUNIOR, 2014, p. 212).

\section{COMPETENZA}

La competenza è un elemento fondamentale per la soddisfazione del giusto processo. La difesa dei diritti di solidarietà avviene a favore di una collettività, che può essere separata e senza alcun legame che la unisca, che porta l'attenzione al momento della fissazione della competenza.

Per la fissazione della competenza territoriale nelle richieste collettive, Neves elenca sette passaggi necessari per la divulgazione della sentenza competente. Si sottolinea che la fissazione della competenza territoriale è una conseguenza 
dell'analisi della legislazione procedurale, allo stesso modo in cui viene effettuata nei casi di protezione singolare, poiché si tratta di una questione legata alla legge trattata in modo isonomico alla protezione individuale (NEVES, 2016, pag. 168).

Tuttavia, è necessario sottolineare che la legislazione che disciplina la protezione dei diritti collettivi può stabilire la giurisdizione che ha origine in determinati tribunali o organi atipici, secondo le modalità di ciò che può accadere nel processo collettivo speciale, nell'azione popolare, nell'azione civile pubblica, nell'ingiunzione collettiva, nel mandato collettivo e nell'azione di cattiva condotta amministrativa.

A causa della rilevanza delle misure, vale la pena notare che la competenza nella sfera collettiva è regolata dalle arti. 2, la legge sull'azione civile pubblica, e 93, del codice di tutela del consumo, che stabiliscono come criterio di competenza territoriale il luogo del danno.

Inoltre, a seconda della legislazione del LACP, la competenza sarebbe territoriale e funzionale, il che solleva il dibattito se la competenza sarebbe dovuta al forum, alla questione territoriale, o alla maggiore efficacia e facilità, in virtù del criterio funzionale. Tuttavia, è certo che la competenza territoriale è assoluta, il che porta al suo rispetto obbligatorio.

Dopo aver determinato il foro competente, inizia la ricerca del giudizio competente, che sarà fatto dalle leggi di organizzazione della magistratura e del CPC. Questo perché la competenza della sentenza è influenzata dalla competenza territoriale, mai il contrario (NEVES, 2016, pag. 186).

\section{ESECUZIONE DELLA SENTENZA EMESSA NELL'AMBITO DI AZIONI COLLETTIVE}

La base per l'esecuzione di una sentenza individuale è indossata dall'adempimento di un giudizio collettivo, che deve avvenire come una continuazione del processo di conoscenza, dopo il momento in cui il debitore non ha rispettato il comando della 
sentenza. La fase di attuazione sarà rispettata mediante l'esecuzione di una sentenza, secondo le circostanze previste dall'articolo 515 del CPC.

In relazione ai diritti diffusi e collettivi stricto sensu la tutela del diritto materiale sarà fatta attraverso l'esecuzione collettiva, che si svolgerà a beneficio della comunità, che sarà fatta attraverso gli aspetti utilizzati nell'esecuzione individuale, senza alcuna specialità procedurale dovuta alla natura collettiva di interesse (NEVES, 2016, p. 392-393).

L'esecuzione può dar luogo ad un'ingiunzione (divieto di illegittimità) o in natura risarcitoria, come il risarcimento del danno, o in denaro, che, in tal caso, deve essere restituito alla persona giuridica di diritto pubblico che ha subito il danno 0 al il Fondo dei diritti diffusi (art. 13 della legge federale n. 7347/1985) (DIDIER JÚNIOR; ZANITI JÚNIOR, 2014, p. 412).

Per quanto riguarda la legittimità, qualsiasi parte integrante del polo attivo può promuovere l'esecuzione, anche se non ha partecipato alla fase cognitiva, secondo l'interpretazione dell'articolo 15 della legge 7.347, che elenca come requisito il lasso di tempo di 60 giorni del transito finale in modo che nuovi soggetti partecipino al processo ai fini dell'avvio della fase di attuazione (DIDIER JÚNIOR; ZANETI JÚNIOR, 2014, p. 413). Cioè, l'esecuzione collettiva dei diritti diffusi o collettivi stricto sensu può essere fatta da qualsiasi straordinario legittimato, rispettando il corso temporale del transito finale. Ciò è previsto anche dall'articolo 15 del CBPC-IBDP e dall'articolo 26 del CBPC-UERJ/UNESA.

Si sottolinea l'obbligo di proporre l'adempimento del giudizio come attribuzione essenziale al parquet, nella cura degli interessi collettivi (NEVES, 2016, p. 391).

Tuttavia, vale la pena sottolineare che l'obbligo del Pubblico Ministero di eseguire una sentenza in azione civile pubblica sorge dopo 60 giorni dalla sentenza definitiva della sentenza, ai sensi dell'articolo 15, della legge 7.347/85. 
In relazione all'azione popolare, il dovere della Procura di eseguire la pena è pendente quando il cittadino è inerte per più di 60 giorni, conteggiati dalla pubblicazione della sentenza di secondo grado, ai sensi dell'art.16, della Legge 4.717/65. Cioè, ci sarebbe il dovere funzionale del parquet mobile di rispettare una frase provvisoria, prima della possibilità di modifica del comando della frase.

Pertanto, c'è anche la possibilità di un'applicazione individuale nell'esecuzione dei diritti diffusi e collettivi stricto sensu, secondo la lezione di Didier:

É também possível que a sentença proferida em um processo em que se discutem direitos difusos ou coletivos seja utilizada por um indivíduo corno título de uma execução individual, tendo em vista a extensão in utilibus da coisa julgada coletiva ao plano individual, já examinada. Obviamente, antes de executar a decisão, o indivíduo deverá proceder à liquidação do seu crédito, em que deverá demonstrar, inclusive, que é titular de um crédito (liquidação com thema decidendum mais amplo do que a liquidação individual prevista no CPC, restrita à decisão sobre o valor ou sobre a coisa a ser executada) (DIDIER JUNIOR; ZANETI JUNIOR, 2014, p. 415).

Per quanto riguarda i diritti individuali omogenei, che sono diritti individuali aggregati dalla loro origine comune, l'esecuzione sarà effettuata individualmente dai titolari. Cioè, la forte componente di collettività che esisteva nella fase di conoscenza è rallentata nella fase di esecuzione, poiché l'interesse individuale sarà trattato come collettivo nella fase di esecuzione (NEVES, 2016, p. 394). Per quanto riguarda i forum, l'articolo 516 del CPC impone una norma rigorosa, un criterio di competenza assoluta, che porterebbe all'esecuzione di una sentenza per la sentenza che ha deciso la causa nel primo grado di competenza.

L'interpretazione di tale disposizione di legge include l'esecuzione individuale di una sentenza collettiva, tuttavia, in base a criteri di praticità, Neves (2016, pag. 376) difende come competente per la risoluzione individuale del giudizio collettivo il foro del domicilio del liquidatore, cosicché non vi è una grande concentrazione di procedimenti nel distretto che ha deciso la causa in primo grado di competenza e meno oneri per l'esecutore. 
Tuttavia, va rilevato che vi è stato un veto presidenziale in relazione al singolo comma dell'articolo 97 del CDC, che esclude l'ipotesi che il foro di esecuzione di una sentenza collettiva sia scelto come quello del domicilio dell'esequinte.

Secondo Zavascki (2005, p. 164), non c'è modo di considerare inutile il veto, poiché apre la strada all'interpretazione e rivela ragioni che dovrebbero essere considerate, poiché considerate dai legislatori.

Tenuto conto della determinazione espressa della competenza per l'esecuzione della decisione, è quella della decisione che ha giudicato la causa nel primo grado di competenza, ai sensi dell'articolo 516, II del CPC, non sussistono dubbi sulla competenza.

98 del CDC, in relazione alla possibilità della Procura della Repubblica e degli enti pubblici (legittimati dall'art. 82 del CDC) di promuovere l'esecuzione di interessi individuali omogenei, poiché la sfera collettiva si svuota al momento dell'esecuzione di interessi individuali. Evidenziando la lezione di Zavascki:

Quanto ao Ministério Público e órgãos públicos, não se vislumbra circunstância na qual tais entes teriam algum interesse em promover liquidação e execução específicas em favor de particulares, ainda mais mediante representação. Em consequência, é certo não possuir - Ministério Público legitimidade para promover o tratamento individualizado da sentença genérica, mas não porque ele não tem poderes para agir em nome próprio em favor das vítimas e sucessores e sim porque, nessa hipótese, não se trata de defesa de interesses sociais ou individuais indisponíveis (ZAVASCKI, 2005, p. 163).

Infine, è interessante notare che anche coloro che non fanno parte del processo possono lamentarsi di quanto proposto in un giudizio collettivo, poiché il suo scopo è quello di dare concretezza ai diritti della comunità:

Diante disso, os instrumentos processuais utilizados para tutela dos interesses transindividuais devem ser adaptados à natureza do interesse substancial em questão, de modo a lhe proporcionar uma maior efetividade. Para tanto, algumas alterações legislativas foram feitas com a finalidade de atender esse escopo. Dentre elas menciona-se a tratativa dada à sentença coletiva, à coisa julgada e à execução de sentença coletiva. A sentença coletiva, dada

RC: 103912

Disponibile in: https://www.nucleodoconhecimento.com.br/legge/diritti-di-solidarieta 
sua natureza, possui um alcance diferenciado no que se refere aos indivíduos por ela albergados, podendo atingir, inclusive, aquele que não foi parte no processo, dada a extensão secundum eventum litis da coisa julgada coletiva ao plano individual (ALFREDO, BALEOTI, 2012, p. 435).

\section{GUARDIANI DELL'URGENZA NELLE AZIONI COLLETTIVE}

In generale, la protezione dell'urgenza nelle azioni collettive non presenta divergenze di ragionamento in relazione alle azioni individuali (DIDIER JÚNIOR; ZANETI JÚNIOR, 2014, p. 342), che porta l'incidenza del CPC quando ci sono i requisiti stabiliti nel suo articolo 300 .

Tuttavia, ci sono alcune procedure speciali che prevedono questa tecnica procedurale, come l'articolo 12 del LACP, che consente di garantire una protezione precoce nella difesa di determinati interessi collettivi (DIDIER JÚNIOR; ZANETI JÚNIOR, 2014, p. 342).

A titolo di esempio, inoltre, l'articolo 4 del LACP prevede la possibilità di misure ingiuntive con lo scopo di prevenire danni ai beni giuridici ivi descritti. Giustamente, la Didier conduce alla correzione delle disposizioni di tale articolo, in quanto la misura sarebbe soddisfacente, in quanto concederebbe il diritto richiesto attraverso la provocazione del potere giurisdizionale, non una misura cautelare:

Embora mencione expressamente a tutela cautelar, a redação do dispositivo não dá margem a dúvida: não se trata de tutela cautelar, mas, sim, tutela inibitória, que é satisfativa e visa exatamente obter providência judicial que impeça a prática de ato ilícito e, por consequência, a ocorrência de um dano (DIDIER JÚNIOR; ZANETI JÚNIOR, 2014, p. 343).

È importante evidenziare alcuni impedimenti alla concessione della protezione provvisoria nelle azioni collettive, come le disposizioni dell'articolo 2 della legge federale n. 8.437/1992 e del $\S 2$ dell'articolo 22 della legge n. 12.016/2009, che limitano la concessione di un'ingiunzione in un mandato di garanzia collettiva 0 in un'azione civile pubblica all'audizione del rappresentante giudiziario della persona giuridica di diritto pubblico, deve essere effettuato entro un periodo massimo di 72 ore.

RC: 103912

Disponibile in: https://www.nucleodoconhecimento.com.br/legge/diritti-di-solidarieta 
Inoltre, è simile a quanto avviene per la concessione di un'ingiunzione nella controversia collettiva per il possesso di beni, che è subordinata all'adempimento dei requisiti di cui all'articolo 565 del codice di procedura civile.

\section{RICARABILITÀ NELLE AZIONI COLLETTIVE}

Non ci sono mezzi specifici di ricorso per l'uso nelle azioni collettive. Ci sono menzioni eccezionali di impugnabilità nell'applicazione nelle domande collettive, che porta all'applicazione generale del sistema di ricorso del codice di procedura civile (NEVES, 2016, p. 324)

Quanto all'impugnazione della decisione cautelare, l'art. $1.015 \mathrm{cpc}$ elenca l'elenco delle ipotesi che danno luogo al deposito di un ricorso interlocutorio. Per espressa autorizzazione del predetto articolo, al suo capo XIII, gli altri casi previsti dalla legge consentono la trattazione del ricorso cautelare, in cui l'art. 19, § 1, della Legge $4.717 / 65$, con la previsione che un ricorso cautelare è possibile a causa di una decisione cautelare in una class action.

Secondo Neves (NEVES, 2016, p. 325), a causa del microsistema collettivo, la suddetta norma dovrebbe essere applicabile in tutte le altre azioni collettive, il che consentirebbe l'interposizione di un danno strumentale in qualsiasi decisione interlocutoria sorta in un processo collettivo.

Per quanto riguarda la legittimità ricorsiva, è interessante analizzare che esistono eccezioni alla regola del 996 del CPC, che stabilisce la parte soccombente, i terzi interessati e i pubblici ministeri come beni legittimi ai ricorsi.

Va sottolineato che l'articolo 996 del CPC riguarda l'interesse ricorsivo e non la legittimità ricorsiva, poiché, quando si tratta di una parte fallita, viene effettuata un'analisi del soccombente, che porta a un'analisi concreta della decisione che mette in discussione l'interesse ricorsivo (NEVES, 2016, p. 330). La legittimità non ha nulla a che fare con l'interesse, perché è una parte legittima ricorrere a chi vince o perde, il 
criterio di stabilire la legittimità è totalmente indipendente dal contenuto della decisione.

Vale la pena sottolineare un tema importante: secondo la regola dell'articolo $19, \S 2$, della legge 4.717/65, ogni cittadino può impugnare le sentenze e le decisioni emesse a seguito di un'azione popolare. Risulta che, a seconda della regola di cui all'articolo 996 del CPC, il cittadino che non compone il polo attivo del corso non rientra come parte soccombente o terzo, poiché è diffuso il diritto di commento nell'azione popolare, il che rimuove il concetto di terzo interessato al solo paragrafo dell'articolo 996, CPC. Pertanto, il cittadino non compone l'elenco dei soggetti che hanno legittimità ricorsiva, ma trova il permesso nel LAP di fare appello.

Per quanto riguarda gli effetti ricorsivi (ostinati, tralattivi, espansivi, sostitutivi, regressivi e differiti) non c'è particolarità quando generati nel processo collettivo. Solo per quanto riguarda l'effetto sospensivo, è necessario esaminare la normativa relativa a ciascuna azione, al fine di verificare l'assenza o la presenza di ciascun effetto ricorsivo di cui trattasi (NEVES, 2016, pag. 332).

Vale la pena sottolineare la normativa che disciplina l'Azione Civile Pubblica, che prevede per il giudice un effetto sospensivo sui ricorsi con la finalità di distogliere il danno irreparabile alle parti, a seconda dell'articolo 14 della legge 7.347/1985, che ha costituito la conclusione che i ricorsi presentati nell'ambito dell'Azione Pubblica Civile non hanno effetto sospensivo solo a causa della loro mera interposizione (NEVES, 2016, p. 332). Pertanto, il ricorso, che, come regola generale, ha effetti sospensivi, in forza dell'articolo 1.012 del codice di procedura civile, deve rispettare i requisiti elencati nell'articolo 14 del LACP (evitando danni irreparabili a parte) per evitare che la sentenza emessa nell'ambito dell'azione civile pubblica produca effetti immediati.

\section{CONSIDERAZIONI FINALI}

Alla luce della discussione svolta da questo lavoro, è possibile osservare che il testo del CRFB/88 acquista forza ed efficacia con la protezione dei diritti collettivi, alla 
maniera di una ricerca per la materializzazione delle garanzie sociali che sono previste nel vernacolo costituzionale e possono essere rivendicate giudizialmente.

Tale strumento è estremamente interessante per la giustizia sociale da realizzare in una società di massa, dove la violazione di un diritto può andare oltre la sfera individuale e raggiungere una collettività di persone. Per questo motivo, al fine di rispondere al problema presentato inizialmente, il pregiudizio costituzionale di garantire i diritti di solidarietà è stato evidenziato conferendo unicità alle disposizioni giuridiche, che cercano di garantire diritti collettivi in confluenza al raggiungimento del principio di giustizia sociale e giusto processo.

Esplorando gli elementi essenziali della protezione collettiva, è stato presentato un sistema armonico e che trova coerenza per garantire la tutela del bene giuridico di tutta la collettività. Così, il problema dell'ermetismo di questo sistema procedurale è stato superato, in quanto è stato affrontato in modo tale da discutere la sua matrice costituzionale e le sue basi giuridiche. Pertanto, ciò che potrebbe assomigliare a un sistema disconnesso, perché ha standardizzazione in varie legislazioni, viene interpretato come uno strumento integrato che si muove per garantire diritti che appartengono non a una sola persona, ma alla società.

\section{RIFERIMENTI}

ALFREDO, Luciana Romaneli Rodrigues e BALEOTTI, Francisco Emílio. A sentença coletiva e a efetividade dos direitos transindividuais. Revista Jurídica Cesumar, Vol. 12, No 2, 2012.

BELLINETTI, Luiz Fernando; SOUZA, Raíssa Fabris. Obstáculos para a efetividade da tutela de direitos transindividuais por entidades sindicais em prejuízo ao efetivo acesso à justiça. Revista Cidadania e Acesso à Justiça, Florianópolis, SC, v. 5, p. 1-17, 2019.

DIDIER JUNIOR, Fredie; ZANETI JUNIOR, Hermes. Curso de Direito Processual

Civil: processo coletivo. 9. ed. Salvador: JusPodivm, 2014. 
HACHEM, Daniel Wunder. A dupla titularidade (individual e transindividual) dos direitos fundamentais e econômicos, sociais, culturais e ambientais. Revista de Direitos Fundamentais e Democracia, v. 14, n. 14, Curitiba, p. 618-688, jul./dez. 2013.

NEVES, Daniel Amorim Assumpção. Manual de processo coletivo. São Paulo: Método, 2016.

SANTOS, Enoque Ribeiro dos. Processo coletivo do trabalho. 2. ed., rev., atual. e ampl. Rio de Janeiro: Forense, 2018.

ZAVASCKI, Teori Albino. Processo coletivo: tutela de direitos coletivos e tutela coletiva de direitos. Tese (Doutorado Programa de Pós-graduação em Direito) Faculdade de Direito da Universidade Federal do Rio Grande do Sul, Porto Alegre, 2005.

Inviato: Dicembre, 2021.

Approvato: Dicembre, 2021. 